\title{
Embedded B-spline Reconstruction in the Volume Rendering
}

\author{
Zeljka Mihajlovic, Leo Budin, IEEE Member \\ Department of Electronics, Microelectronics, Computer and Intelligent Systems, \\ Faculty of Electrical Engineering and Computing \\ Unska 3, 10000 Zagreb, Hrvatska \\ e-mail: zeljka.mihajlovic@fer.hr, leo.budin@fer.hr \\ phone: +38516129 944; fax: +38516129653
}

\begin{abstract}
This paper deals with reconstruction problem in the visualization. The representation of the object is based on the volumetric data. Volumetric data is sampled on a regular grid. The surface of the object is implicitly defined as a zero value of the volumetric function. In the volume rendering procedure, intersection of the ray with the object is required. Trilinear interpolation is usually used for the reconstruction.

In this paper we propose interpolation B-spline embedded directly into the rendering procedure. Different artifacts may appear due to the reconstruction in the rendering procedure. If the quality of the rendered object is of the crucial importance, we have to pay attention to the reconstruction in the rendering procedure.
\end{abstract}

Keywords: visualization, interpolation B-spline, reconstruction, volume rendering

\section{INTRODUCTION}

In almost all visualization methods the problem of conversion between continuous and discrete representation appears. If we did not pay attention to this conversion alias artifacts will appear. Volumetric space consists of volume elements. Each volume element may represent result from real world object sample, numeric simulations, or may represent some pure mathematical value. The samples are taken from continuous space, but object with sharp edges in that space creates discontinuity. According to Shannon theory, signal can be reconstructed from its samples if two conditions are valid. First, spectrum of the signal must be bandlimited, and sampling frequency must be twice higher than the largest frequency present in the signal. The alias that occurs during sampling stage is called prealias and postalias is caused by the reconstruction.

Different scanners can achieve data acquisition: CT (Computer Tomography) or MR (Magnetic Resonance), for example. During the sampling process some lowpass filtering is performed, but information about it for sequences of slices available on Internet, is usually unknown. If the sampling is not done correctly, information can be irrecoverable lost.

The resolution of scanned slices is usually high, but number of slices is often insufficient because of radiation risks for patient. To enlarge the number of slices, interpolation between the slices is required. Compression of the volume data is also desired because the size of data set is large. Thus the reconstruction of the compressed volume, interpolation between slices, or interpolation of the volume elements becomes important step.

There are many visualization techniques, and common to all of them is necessity of the reconstruction. Polygonal mash, for some visualization techniques, has to be reconstructed. For example, in Marching cubes [1], positions of the polygon vertices should be determined. Only linear interpolation between two neighboring voxels for this determination is usually used. The "splatting" methods of Westover [2] assume a truncated Gaussian filter for the interpolation. Volume rendering that uses 3D texture maps relays on the hardware implementation of resampling. 


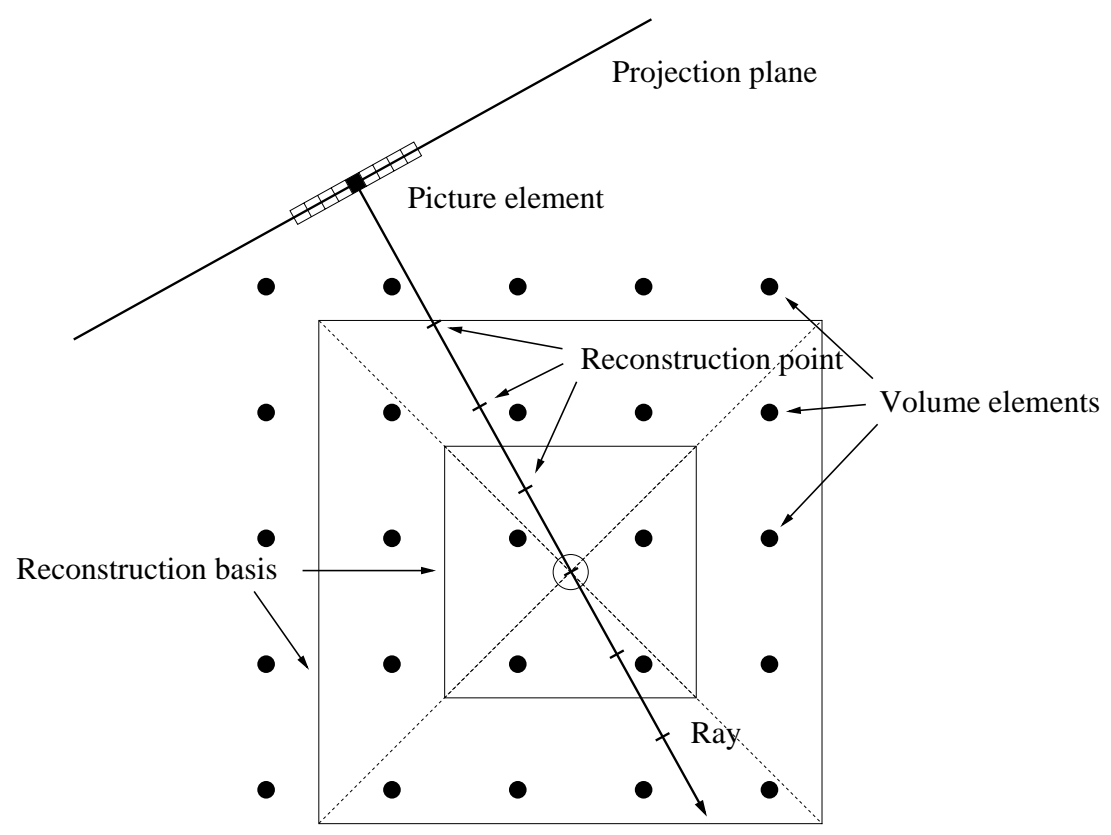

Figure 1. Reconstruction in the volume rendering.

Fourier volume rendering [3] requires reconstruction of the cutting plain trough the 3D frequency domain at arbitrary angle, and it is very delicate step. For this purpose POCS technique is used. In the volume rendering rays are cast in the volume element space and reconstruction is performed along each ray at many positions. For this reconstruction trilinear interpolation is often used. Similarly, shear warp factorization technique [4] uses combination of the two bilinear interpolations. Typical appearance of alias due to the reconstruction scheme are: terrace appearance of the surface, loss of the fine details (blurring), artificial cracks on the surface, ghost parts of the object.

To achieve better interpolation than trilinear, Mitchell and Netravali introduced BC-splines [5]. Marschner and Lobb [6] made further investigation of the BC-splines. In order to verify the reconstruction, they proposed error metrics for each of the reconstruction artifacts. Moller, Machiraju, Mueller and Yagel [7] found that, in the class of the BC-spline filters, the Catmull-Rom spline and its derivative give the most accurate reconstruction. In order to analyse, classify and estimate the error of the applied filters, they used Taylor Series expansion of the convolution sum $[8,7]$.

In reconstruction, it is important to distinguish approximation and interpolation approach. The approximation curves are used to approximate the control polygon, and interpolation curves must pass through the defined vertices. Hence, convolution with reconstruction filter can produce approximation or interpolation of the volume elements. The BC-splines are mostly approximation splines. Unser propose B-spline transform as prefilter required for interpolation B-spline $[9,10]$. They use direct and indirect B-spline transform to achieve efficient mechanisms for scaling and resampling of the image.

\section{VOLUME RENDERING}

One of the most popular visualization method is volume rendering $[11,4]$. In the volume rendering rays are cast in the volume element space. Volume element space is discrete representation of the sampled data. During the volume rendering there are several layers where reconstruction is necessary, and the error caused by reconstruction may occur. Reconstruction is done in the three-dimensional space based on the values of the volume elements. We must be able to interpolate the function at arbitrary locations to obtain the volume densities. Numeric integration along the ray path uses reconstructed values at sample points. To calculate value assigned to the ray, values at the sample points along the ray are accumulated. Final reconstruction is done based on each ray in order to produce the final image (Fig. 1).

Usual approach is trilinear interpolation based on the eight neighbors. For trilinear interpolation four volume elements around the circled reconstruction point, in the cross section in the volume element space, are included 


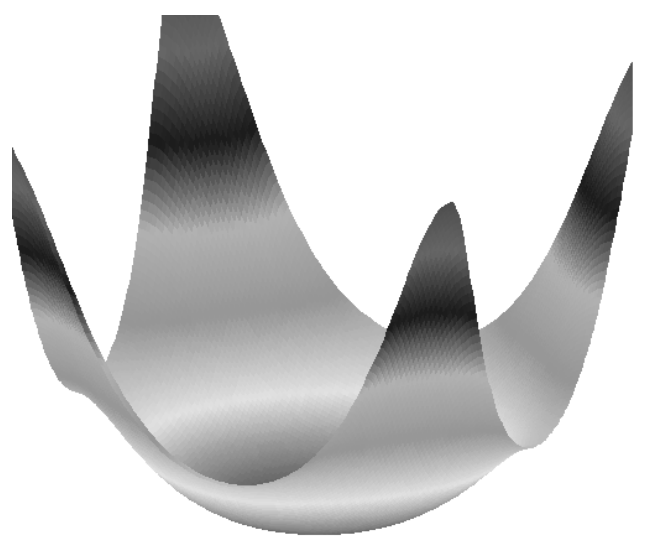

Figure 2. Prefilter for the cubic interpolation B-spline.

(cf. Fig. 1). Better interpolation include larger reconstruction base. Spot where reconstruction is required in general case does not match the grid point. Therefore, reconstruction of the continuous function in the volumetric space is required.

There are two approaches to achieve better quality of the reconstructed object. First approach is to interpolate samples in the volume element space on regular grid and second is to use the better reconstruction along the ray. Hybrid approach is also possible: to increase volume element space with more quality interpolation and than reconstructs the value on the ray with trilinear interpolation.

For interpolation B-spline reconstruction kernel is too wide, so well as sinc reconstruction kernel. But, if prefiltering is applied for cubic interpolation B-spline, the size of the reconstruction kernel is acceptable.

\section{INTERPOLATION B-SPLINE}

B-splines of order $n$ are piecewise polynomial functions of degree $n$. Any continuous $n$-th degree polynomial piecewise function can be represented using B-spline functions of the same order. In the case of uniform spacing between knot points, such a function can be represented in the form:

$$
f_{n}(x)=\sum_{k=-\infty}^{\infty} c_{n}(k) \beta_{n}(x-k),
$$

were $\beta_{n}(x)$ is $n$-th order B-spline weight function. Sequence $c_{n}(k)$ denotes B-spline coefficients. If we use volume elements for coefficients $c_{n}(k)$, function $f_{n}(x)$ presents approximation B-spline for the given set of points. The B-spline is separable function, so convolution could be separately applied in each space direction. For the interpolation B-spline, function $f_{n}(x)$ is known at the set of discrete points. The values of the function $f_{n}(x)$ are the volume elements, and $c_{n}(k)$ should be determined.

Fourier transform of identity (1) gives:

$$
F(\omega)=C_{n}(\omega) B_{n}(\omega)
$$

where $F(\omega), C_{n}(\omega)$ and $B_{n}(\omega)$ are Fourier domain representations of $f(x), c_{n}(x)$ and $\beta_{n}(x)$ respectively. Fourier transform of $c_{n}(x)$ easily can be derived from (2) in the form:

$$
C_{n}(\omega)=\frac{1}{B_{n}(\omega)} F(\omega) .
$$

This equation is valid only if $B_{n}(\omega)$ has no zeroes on the frequency axis $\omega$, and this is satisfied for all B-splines. The frequency domain representation of B-spline prefilter is:

$$
P_{n}(\omega)=\frac{1}{B_{n}(\omega)},
$$



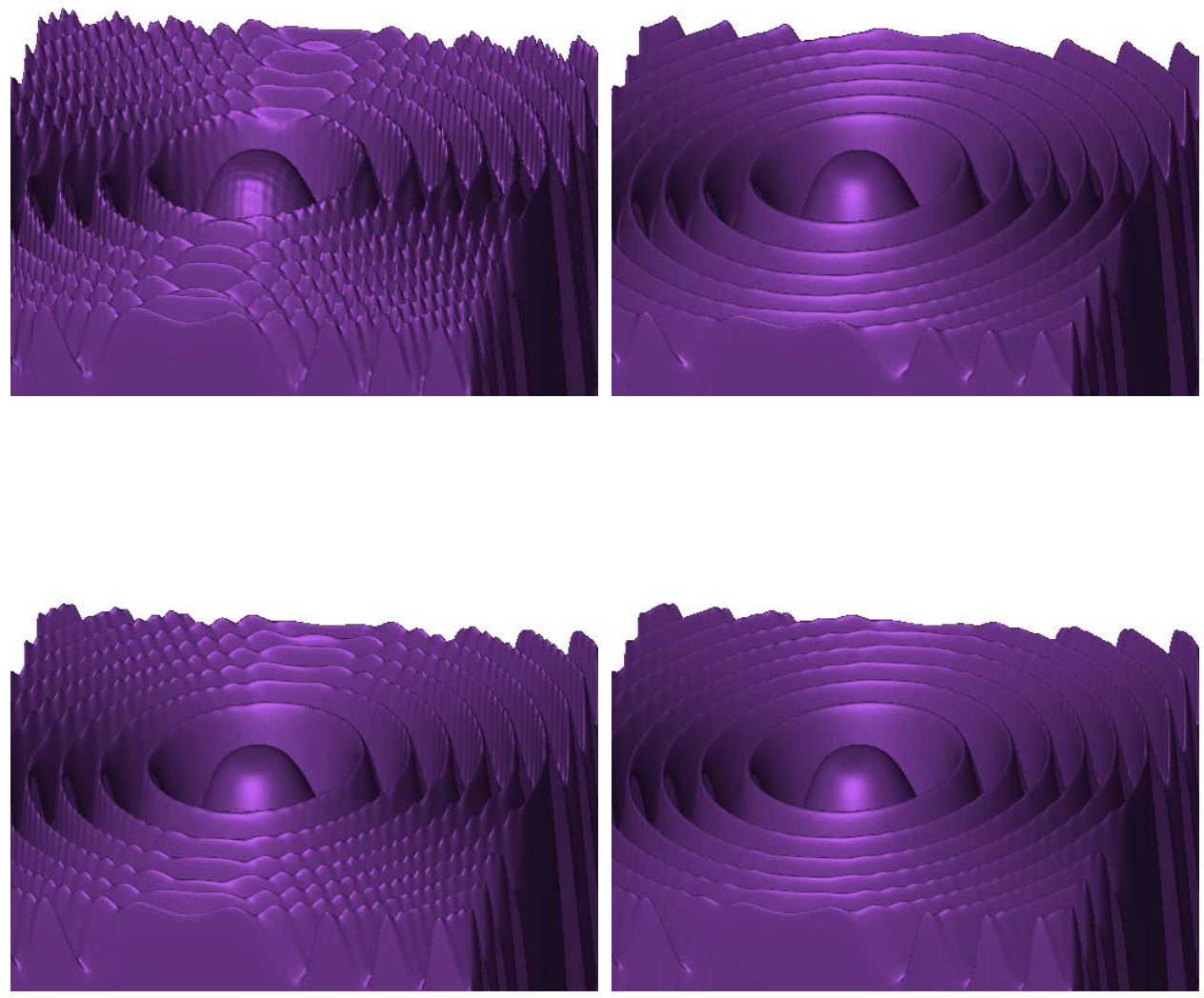

Figure 3: First row shows triliner reconstruction (left) and approximation B-spline (right). Second row shows CatmullRom spline (left) and interpolation B-spline (right).

where denominator is:

$$
B_{n}(\omega)=\beta_{n}(0)+2 \sum_{k=1}^{\left\lfloor\frac{n}{2}\right\rfloor} \beta_{n}(k) \cos (k \omega) .
$$

Operator $\lfloor\cdot\rfloor$ denotes the largest integer value not greater than its argument. For example, prefilter for the cubic interpolation B-spline is:

$$
P_{3}(\omega)=\frac{3}{2+\cos (\omega)}
$$

Figure 2 shows the frequency domain representation of the prefilter from the equation (6), as twodimensional case (DC is in center of the shown function). Similar function is in the volumetric space.

\section{EMBEDDED INTERPOLATION B-SPLINE}

Interpolation B-spline requires determination of the spline coefficients. This step is direct B-spline transform. Direct B-spline transform could be done in the space domain or in the Fourier domain. After direct B-spline transform the volume element space is prepared for interpolation. We implement B-spline reconstruction kernel 
directly in the volume rendering procedure. Reconstruction is performed along the ray in the transformed volume element space.

Figure 3 shows images generated with four reconstruction kernels. Trilinear reconstruction exhibit strong alias artifacts. Catmull-Rom interpolation spline is wide as B-spline reconstruction kernel, but artifacts are still there. Approximation B-spline produces smooth result. The problem with approximation B-spine is smoothing. The waves are shallow and sharp parts are lost. For interpolation B-spline, direct B-spline transform is applied on the volume element space. Rendering is performed on the transformed space and the most pleasant result is achieved.

\section{CONCLUSION}

Reconstruction in the volume rendering procedure is important step because it could provokes strong alias artifacts. Terrace appearance of the surface, loss of the fine details (blurring) and artificial cracks on the surface or ghost parts of the object may introduce incorrect interpretation about the object. The proposed interpolation Bspline reconstruction procedure, directly implemented in the volume rendering procedure, produces high quality rendering tool.

A comparison to usual reconstruction methods confirms our result. Besides the volume rendering, there are many visualization techniques where reconstruction is delicate step. Proposed reconstruction could be adapted to thise techniques also.

\section{References}

[1] W. E. Lorensen and H. E. Cline, "Marching cubes: A high resolution 3d surface construction algorithm," Computer Graphics, vol. 21, pp. 163-169, July 1987.

[2] L. Westover, "Footprint evaluation for volume rendering," Computer Graphics, vol. 24, pp. 367-376, August 1990 .

[3] T. Malzbender, "Fourier volume rendering," ACM Transactions on Graphics, vol. 12, pp. 233-250, July 1993.

[4] P. Lacroute and M. Levoy, "Fast volume rendering using a shear-warp factorization of the viewing transformation," Computer Graphics, vol. 28, pp. 451-458, July 1994.

[5] D. P. Mitchell and A. N. Netravali, "Reconstruction filters in computer graphics," Computer Graphics, vol. 22, pp. 221-228, August 1988.

[6] S. R. Marschner and R. J. Lobb, “An evaluation of reconstruction filters for volume rendering," Proc. Visualization '94, IEEE CS Press, pp. 100-107, October 1994.

[7] T. Moller, R. Machiraju, K. Mueller, and R. Yagel, "Evaluation and design of filters using a taylor series expansion," IEEE Transactions on Visualization and Computer Graphics, vol. 3, pp. 184-199, June 1997.

[8] R. Machiraju and R. Yagel, "Reconstruction error characterization and control: A sampling theory approach," IEEE Transactions on Visualization and Computer Graphics, vol. 2, pp. 364-376, December 1996.

[9] M. Unser, A. Aldroubi, and M. Eden, "B-spline signal processing:part i-theory," IEEE Transactions on Signal Processing, vol. 41, pp. 821-833, February 1993.

[10] M. Unser, A. Aldroubi, and M. Eden, "B-spline signal processing:part ii-efficient design and applications," IEEE Transactions on Signal Processing, vol. 41, pp. 834-848, February 1993.

[11] M. Levoy, "Display of surfaces from volume data," Proc. IEEE Computer Graphics and Applications, vol. 8, pp. 29-37, May 1988. 